\title{
Multiple Pseudoaneurysms of the Brachiocephalic Artery Following Penetrating Arrow Injury in the Neck
}

\author{
KS Islam ${ }^{1}$, GM Mokbul ${ }^{2}$, MA Quashem ${ }^{1}$, SAMA Sabur ${ }^{1}$, I Zulkarnine ${ }^{1}$, AK Beg $^{3}$, , WZ Bhuyan ${ }^{4}$, Z \\ Alom $^{1}$, I Ahmed ${ }^{1}$, MR Idris ${ }^{1}$, MK Hasan ${ }^{1}$, AR chowdhury ${ }^{1}$ \\ ${ }^{1}$ Department of Cardiac Surgery, NICVD, ${ }^{2}$ Department of Vascular surgery, NICVD, ${ }^{3}$ Department \\ of Anaesthesiology, NICVD, ${ }^{4}$ Department of Radiology and Imaging , NICVD, Dhaka..
}

\begin{abstract}
:
Key words: Arrow injury, Brachiocephalic artery, Pseudoaneurysm, Airway obstruction

Arrow injury in the neck with subsequent pseudoaneurysm formation of the brachiocephalic artery is an uncommon type of injury in our country. Initially it was a punctured wound in the neck which was simply repaired. About 13 days after the initial injury patient came back to hospital with severe respiratory distress and backache for which emergency tracheostomy was needed. This simple puncture wound subsequently developed haematoma in the neck and two pseudoaneurysms at distal brachiocephalic artery. CT angiogram was very helpful to confirm the diagnosis. Correct referral to tertiary hospital like National Institute Cardiovascular Diseases (NICVD), prompt diagnosis, definitive treatment of the injury and subsequent aggressive postoperative management saved the life of this young tailor.
\end{abstract}

(Cardiovasc. j. 2014; 7(1): 58-62)

\section{Introduction:}

The brachiocephalic artery (or brachiocephalic trunk or innominate artery) is the first branch of the aortic arch, and soon after it emerges, the brachiocephalic artery divides into the right common carotid artery and the right subclavian artery. The image of brachiocephalic artery is shown below. ${ }^{1}$ Penetrating jnjury to the brachiocephalic artery with a local made arrow is uncommon in our country.

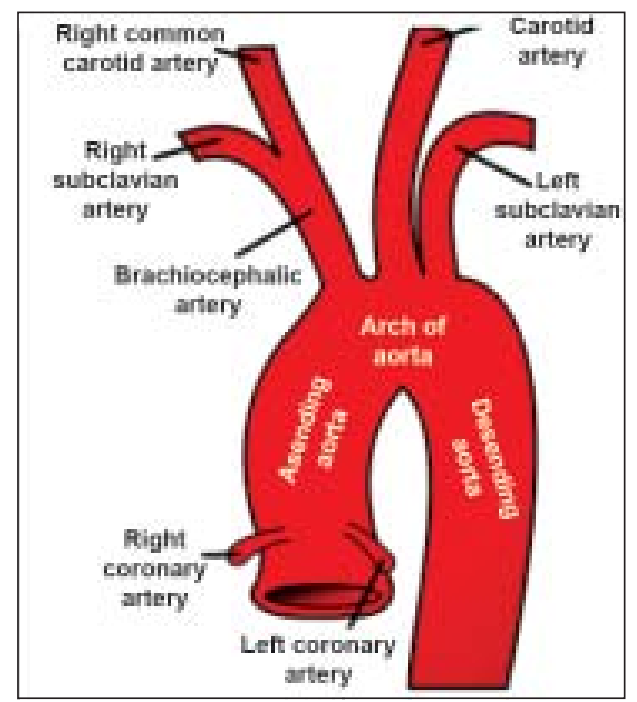

Fig.-1: Aorta and one of its branches brachiocephalic artery.
Our patient is a 22 years old man who sustained an arrow injury in the neck and subsequently developed multiple pseudoaneurysms of the distal brachiocephalic artery. Literature search showed no report of multiple pseudoaneurysms of the brachiocephalic artery following arrow injury. That is why this is unique for case reporting.

\section{Case Report:}

A 22 years old male patient was referred to the department of cardiac surgery from the emergency department of NICVD on $29^{\text {th }}$ October 2013 at 2:20 PM with a suspicion of pseudoaneurysm of the ascending aorta. He had an emergency tracheostomy at Dhaka Medical College Hospital (DMCH) for severe respiratory distress using an endotracheal tube. Attendants told that the patient sustained a penetrating trauma by a locally made arrow at right supraclavicular region near the sternal end on 16.10.2013. He was a bystander of a local dispute at that time. The arrow was then removed with the help of his family members and other peoples. After that there was profuse bleeding from the wound. He was taken immediately to local hospital. Bleeding was stopped by surgical stitching of the skin wound. He also received antibiotics and analgesics. Following this, patient

Address for Correspondence: Dr. Kazi Shariful Islam, Department of Cardiac Surgery, National Institute of Cardiovascular Diseases, Dhaka, Bangladesh. E-mail: kazishariful_islam@yahoo.com. 
started to feel pain in the upper back and neck on the right side with gradual increase of respiratory distress over 13 days after the injury. Ultrasonography with Doppler flow study of the neck and CT scan of the neck and chest were done which revealed a suspected blood vessel injury in the root of the neck with compression of distal trachea and right principal bronchus

At NICVD at the time of initial evaluation, the patient was lying flat on the trolley, conscious oriented but unable to talk due to endotracheal tube in tracheostomy stoma. He was sweating, tachypnoeic, with raised pulse rate, $\mathrm{BP}$ and temperature. There were features of inflammation around the tracheostomy wound. There was diminished breath sound with more resonant percussion note on the right side of the chest. Subsequently endotracheal tube was changed by an adequate sized $(7.5 \mathrm{Fr})$ tracheostomy tube. Clotted blood came out and breath sound improved on right side. Despite this his respiratory distress did not improve. Ultimately he was put on mechanical ventilation sedated and paralyzed.

His complete blood count (CBC) showed increased white blood cell count and raised ESR, Liver function and kidney functions were normal. Chest X-rays P/A and left lateral view (fig $1 \& 2$ ) showed a homogenious oval shaped shadow in the superior and middle mediastinum close to the trachea and right principal bronchus .Ultrasonogram (fig 3) of root of the neck showed large vascular outpouching in the right side of the root of the neck occupying the superior mediastinum, which is continuous with the adjacent great vessel, giving mosaic pattern after colour Doppler application. Bedside Echo was done which revealed normal cardiac status, no pericardial effusion. CT scan of chest (Fig 4a) was done at $\mathrm{DMCH}$ which revealed a rounded non - homogeneous mass in the superior and middle mediastinum compressing the distal trachea and right principal bronchus. CT angiogram (Fig 4b, Fig 4c) of arch of aorta including upper limb vessels was done at Radiology department of the following morning of admission day, with the patient under hand ventilation sedated and paralyzed. This revealed two pseudoaneurysms at distal bracheocephalic artery one is larger than the other size is about $(3.0 \mathrm{~cm} \mathrm{X} 2.5 \mathrm{~cm} ; 1.0 \mathrm{~cm} \mathrm{X} 1.5 \mathrm{~cm})$ with a haematoma compressing right principal bronchus.

Emergency repair of the pseudoaneurysms were done under general anaesthesia without cardiopulmonary bypass. After median sternotomy, exposure of neck vessels was done by extension of the sternotomy incision upward. Controls were taken by soft rubber slings at proximal brachiocephalic artery, right subclavian artery and mid right common carotid artery. After systemic heparinization shunt was inserted from ascending aorta to right common carotid artery to protect cerebral circulation. Large injury (40$50 \%$ of the circumference) at posteromedial aspect of the upper end of the brachiocephalic artery was repaired with PTFE patch and direct suturing of smaller injury at the origin of right subclavian artery with $5 / 0$ proline suture .Haematoma was evacuated to decompress the distal trachea and right principal bronchus.

Patient remained unconscious for two days under ventilation. Neurologist diagnosed it as ischemic encephalopathy, which was treated as per his advice. Patient regained normal cerebral function and was extubated on $3^{\text {rd }}$ postoperative day. Subsequently patient developed pulmonary, mediastinal and Urinary tract infection. After culture and sensitivity report he was treated with appropriate antibiotics. He was referred to ENT consultant for taking care of tracheostomy wound which subsequently healed nicely. Patient was discharged home with reasonably well condition and advice to come after one month for follow up.

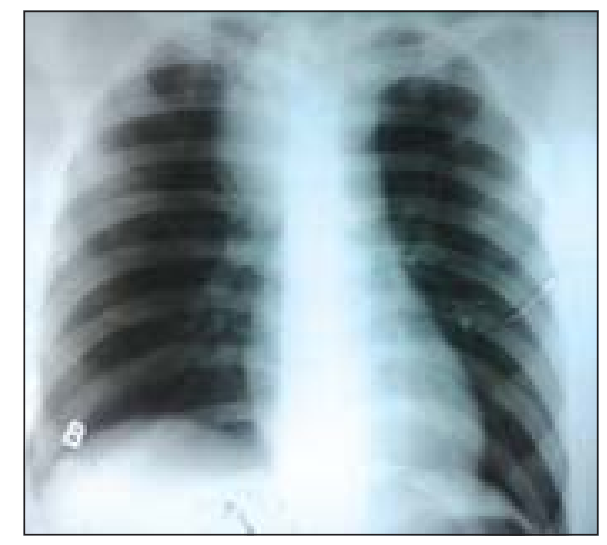

Fig.-2: Chest $x$-ray PA view 


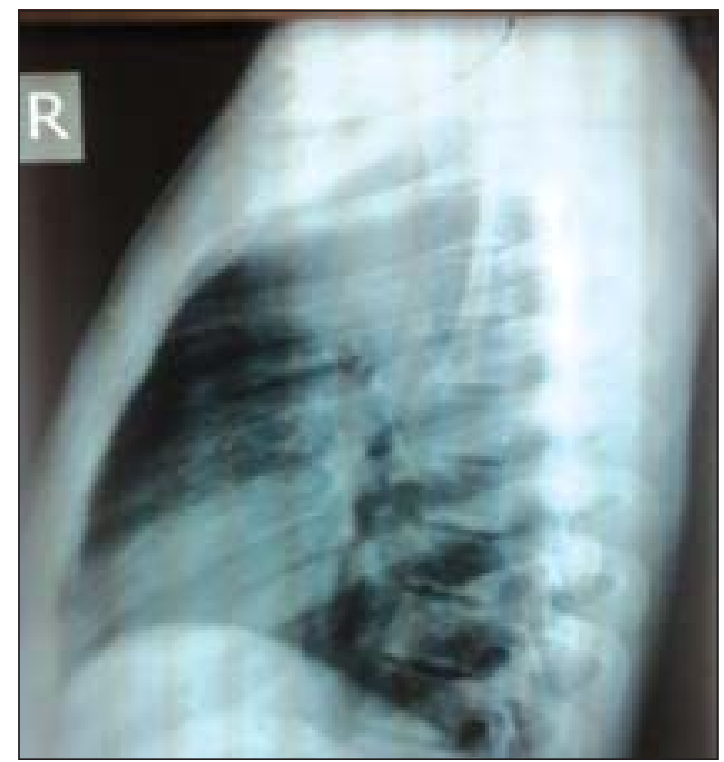

Fig.-3: Chest $x$-ray lateral view



Fig.-4: USG of Neck

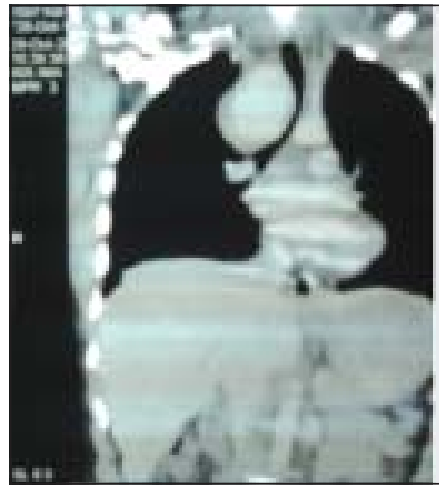

a)

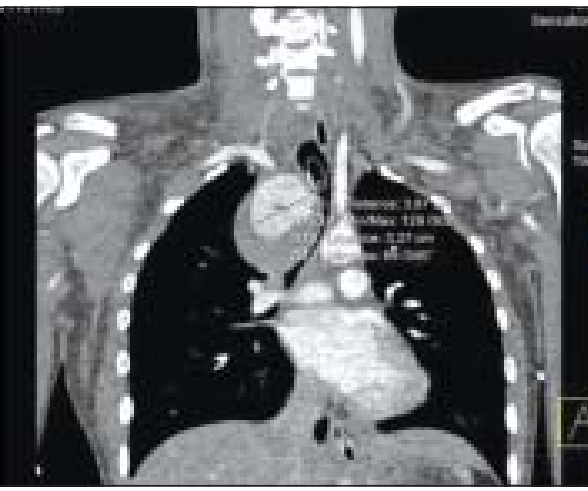

b)

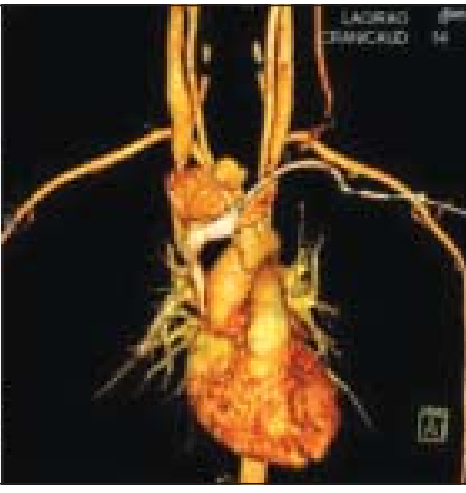

c)

Fig.-5: a) CT scan of the chest (pulmonary window) b) CT Angiogram showing compression of distal trachea and right principal bronchus c) CT Angiogram showing two pseudoaneurysms in the distal Brachiocephalic artery

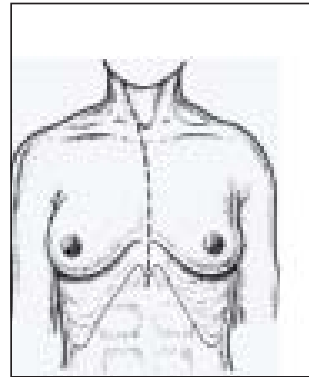

a)

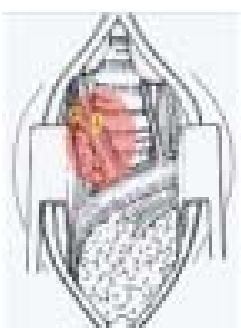

b)

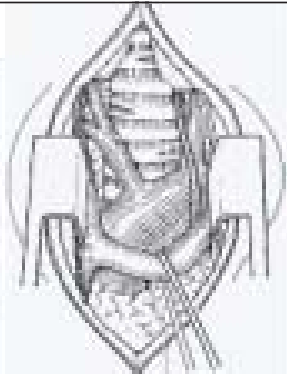

c)

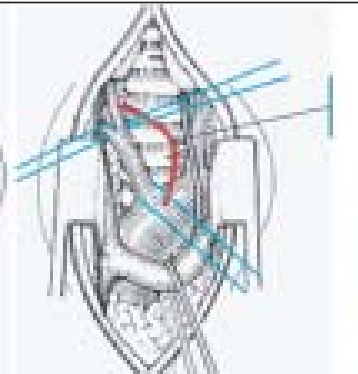

d)

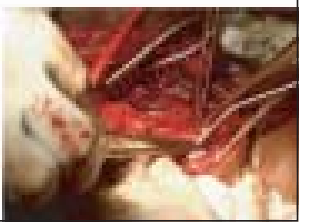

e)

Fig 6: a-e)Diagrammatic \& still pictures showing steps of operation.$^{2(a-d)}$ 




Fig.-7: Patient before discharge

Follow up after one month showed patient improved clinically, trachestomy and chest wound healed nicely, no bruit in the neck.CBC normal, blood culture negative. Duplex study of the neck vessel shows no leakage around the repair.

\section{Discussion:}

The exact incidence of brachiocephalic artery trauma is unknown and it is an uncommon injury. Patients with significant injury die before reaching hospital due to excessive bleeding. ${ }^{3}$ Pseudoaneurysm of this artery following penetrating and blunt trauma have been reported in different articles. ${ }^{3,4}$ Clinical presentations may be different from one patient to other. Some patients may remain asymptomatic for quite a long time after the primary trauma. One such case of stab injury presented 26 years later. ${ }^{5}$ In treated patients mortality may be upto $38 \%$, and increased if multiple vessels are involved. ${ }^{6}$ Usually open surgical repair through median sternotomy with or without cardiopulmonary bypass is done. ${ }^{7}$ Endovascular technique is another option for treatment of such cases. ${ }^{8}$ After confirmation of the diagnosis patients should undergo definitive treatment. To our knowledge multiple pseudoaneurysms of the brachiocephalic artery with compression of right principal bronchus is the first case in the literature where presentation was chest and neck pain associated with severe respiratory distress. Our case had a history of penetrating injury at the neck. After removal of the local made arrow from the neck there was profuse bleeding. It was controlled by giving few stitches at entry point. Patient had the symptom of pain at upper back and neck with gradual onset of respiratory distress. This pain was due to stretching of the surrounding tissues by the expansile haematoma. For his severe respiratory distress, emergency tracheostomy needed. But this tracheostomy did not improve his respiratory distress because it could not relieve distal narrowing. CT scan of the chest showed obvious compression of the lower trachea and right principal bronchus by the haematoma, this finding was discovered later on by us. That was the reason despite tracheostomy, respiratory distress did not improve rather deteriorated. Due to emergency tracheostomy patient developed tissue oedema in the neck and mediastinum. This also contributed further airway compression. Patient needed emergency ventilatory support for severe respiratory distress. Under ventilation CT angiogram was done. This revealed two pseudoaneurysms at distal bracheocephalic artery. Actually CT angiogram gave us the definitive diagnosis and helped us for planning of subsequent surgical management. We performed the operation without cardiopulmonary bypass. After systemic heparinization we used a shunt from ascending aorta to right common carotid artery. After operation patient remained unconscious for $>48$ hours and needed mechanical ventilation. Patient developed ischemic encephalopathy as documented by neuromedicine specialist, he ultimately recovered. Appropriate antibiotic was given according to culture and sensitivity report to combat infection at different sites which patient developed during the course of treatment. He was a young patient with good nutritional status which helped him a lot to overcome various stresses on his body starting from his injury till full recovery.

\section{Conclusion:}

Multiple pseudoaneurysms of brachiocephalic artery with severe respiratory distress is a rare presentation. Clinical evaluation is very important in such case. Penetrating injury in the neck should be investigated properly with appropriate investigations like $\mathrm{CT}$ angiogram to 
rule out any suspected vascular injury. Primary attending physician should have the suspicion and vigilance about the possibility of vascular injury after a penetrating wound in the neck. Any deep puncture wound in the neck should be taken seriously to rule out any major vascular injury which may endanger the life of the patient without proper treatment at proper place. After confirmation of the diagnosis patient should be subjected to definitive treatment as soon as possible at appropriate place. Both surgical and endovascular procedures are available in our country and should be chosen according to the merit of the case.

\section{References:}

1. Brachiocephalic artery picture[image from the internet].2013[updated 2013 March;cited 2014 June 10].Available from: http://en.wikipedia.org/wiki/ Brachiocephalic_artery
2. Bell,P.R.F, Oliver G. Exposure of major blood vessels . In: Carter DC, Russel RCG, Dudley H, Lee G, Eds. Rob \& Smith's operative surgery, Vascular surgery. $5^{\text {th }}$ ed. London: Hodder Arnold 2007. 25

3. Johnston RH Jr, Wall MJ Jr, Mattox KL. Innominate artery trauma: a thirty year experience. J Vasc Surg 1993 Jan;17(1):134-139.

4. Chandler TA, Fishwick G, Bell PR. Endovascular repair of a traumatic innominate artery aneurysm. Eur $J$ Vasc Endovasc 1999;18(1):80-82.

5. Reddi AA, Munasur MM, Naidoo RR, Steer DD. Traumatic innominate artery aneurysm 26 years after stab injury. Ann Thorac Surg 2005;79(3):1034-1036.

6. Hoff SJ, Reilly MK, Merrill WH, Stewart J, Frist WH, Morris JA Jr. Analysis of blunt and penetrating injuries of the innominate and subclavian arteries. Am Surg 1994 Feb;60(2):151-152.

7. Wilson RF. Thoracic vascular trauma. In: Bongard FS, Wilson SE, Perry MO, Eds. Vascular Injuries in Surgical Practice. Connecticut: Appleton \& Lange 1991;107-130.

8. BL. Surgical and endovascular management of penetrating innominate artery injuries. Eur $J$ Vasc Endovasc 2008;36(1):56-62. 